AperTO - Archivio Istituzionale Open Access dell'Università di Torino

\title{
A model for biological control in agriculture
}

\section{This is the author's manuscript}

Original Citation:

Availability:

This version is available http://hdl.handle.net/2318/145462

since

Published version:

DOI:10.1016/j.matcom.2013.02.001

Terms of use:

Open Access

Anyone can freely access the full text of works made available as "Open Access". Works made available under a Creative Commons license can be used according to the terms and conditions of said license. Use of all other works requires consent of the right holder (author or publisher) if not exempted from copyright protection by the applicable law. 


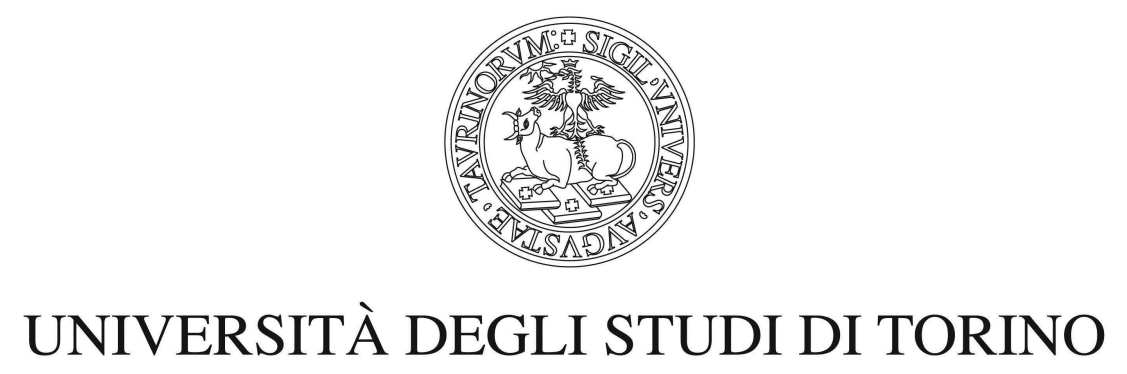

This is an author version of the contribution published on:

M. Sen, M. Banerjee, Ezio Venturino. A model for biological control in agriculture. Mathematics and Computers in Simulation , 87, 30-44, 2013.

DOI: 10.1016/j.matcom.2013.02.001

The definitive version is available at:

http://www.journals.elsevier.com/mathematics-and-computers-insimulation/ 


\title{
A model for biological control in agriculture
}

\author{
M. Sen ${ }^{+}$, M. Banerjee ${ }^{+}$, E. Venturino ${ }^{\dagger}$, \\ ${ }^{+}$Department of Mathematics and Statistics, \\ I. I. T. Kanpur, Kanpur - 208016, India \\ †Dipartimento di Matematica "Giuseppe Peano", \\ via Carlo Alberto 10, Università di Torino, \\ 10123 Torino, Italia \\ Emails: moitri@iitk.ac.in (M. Sen), malayb@iitk.ac.in (M. Banerjee), \\ ezio.venturino@unito.it
}

\begin{abstract}
Persistence and global stability of the coexistence equilibrium of a recently published model in biocontrol of crops are here shown both in absence and presence of delays, introduced to simulate the handling time of the prey. In the latter case, the system can behave in two different ways, in dependence of whether a suitably defined key parameter exceeds a certain threshold. Namely, below the threshold the delay is shown not to be able to influence the stability of the coexistence equilibrium; above it, existence of Hopf bifurcations is analytically proven. Further, in this range, numerical simulations reveal a route to chaotic behavior as function of the size of the delay. Some operative conclusions for agroecosystem management are drawn, although they ultimately depend on each particular situation.
\end{abstract}

Keywords: Global stability, Hopf bifurcations, chaos, spiders, delays

\section{Introduction}

The problem of biological control in agriculture is very important, in view of its economic impact on the minimization of the use of insecticides. In the 
recent literature, it has received a lot of attention, $[11,13,14]$. The role of spiders as generalist predators is of wide interest and well recognized, see for instance [8] and the literature contained in [3]. The use of several spider species for controlling pests in crops as diverse as rice, $[12,16]$, cereals, [1], bananas, [10] is well documented. Mathematical models in this context play an essential role, in that they can substitute costly and lengthy field experiments with rather cheap simulations, using the power of now easily available computational resources.

The purpose of this paper is to revisit a recent paper to improve on its analytic findings, [3]. Specifically, the model considered concerns two spider populations, distinguished because of their living habitats, predating on insects.

In [3], the equilibria have been found and their local stability has been analysed. A delay in transforming the captured prey into predators' newborns has been considered. By means of simulations, the insurgence of Hopf bifurcations has been investigated in terms of the delay parameter.

In this paper, we extend the analytic results, by first concentrating on the non-delayed system, and precisely proving the persistence of and the global stability for it. We then extend the analysis to the delayed model and analytically prove also in this case the persistence, the global stability and the existence of Hopf-bifurcations.

The paper is organized as follows. In the next Section we revisit the basic model. Section 3 contains new results on the non-delayed model, specifically we prove its persistence and the global stability of its coexistence equilibrium. Section 4 contains analogous results for the delay system, and in addition we also prove analytically the existence of Hopf-bifurcations, which in [3] were only discovered numerically.

\section{Background}

In [3] the following three-dimensional system has been considered, modeling an insect population which is hunted by two spider populations. The latter are distinguishable since they reside in two different habitats, in both of which the common prey insects thrive as well. These are represented in general by the cultivated land, whose spider population is then be denoted by $s_{c}$, and the green patches of grass and small woods surrounding the agroecosystem, whose spider population is denoted by $s_{\omega}$. The insect population follows a 
logistic growth law in absence of spiders. Furthermore they represent the only food source for the spider populations. Spiders are subject to natural mortality and in addition they compete for resources.

The ecosystem dynamics is described by the following set of three coupled nonlinear ordinary differential equations.

$$
\begin{aligned}
\frac{d p}{d T} & =r p\left(1-\frac{p}{K}\right)-a p s_{\omega}-b p s_{c} \\
\frac{d s_{\omega}}{d T} & =s_{\omega}\left(e a p-\mu_{\omega}-\frac{s_{\omega}}{W}\right) \\
\frac{d s_{c}}{d T} & =s_{c}\left(e b p-\mu_{c}-\frac{s_{c}}{V}\right)
\end{aligned}
$$

subjected to feasible initial conditions $p(0)>0, s_{\omega}(0)>0, s_{c}(0)>0$.

Here $p(T)$ denotes the insect population while $s_{\omega}(T)$ and $s_{c}(T)$ denote as mentioned the webbuilder spider populations living in two different habitats. The webbuilder spiders are known essentially to live in the same place for their entire lifetime, with the exception of one possible migration known as ballooning, when they are young instars. In fact, it occurs when they release a thread and let themselves be carried by the wind. This phenomenon has been thoroughly analyzed in [17], but here, as in [3], it will be disregarded, in order to concentrate more in the present context on other issues.

All the parameters involved with the model (1) are positive constants. Their ecological interpretation follows: $r$ represents intrinsic growth rate for the insects, with $K$ denoting their environmental carrying capacity. The per capita prey capturing rates are $a$ and $b$ for the spiders in the two habitats. They turn hunted prey into new biomass via the conversion coefficient $0<$ $e<1$. As stated above these spider populations compete with each other intra-specifically, at respective rates $W^{-1}$ and $V^{-1}$. Finally spiders' natural mortality rates are denoted by $\mu_{\omega}$ and $\mu_{c}$.

After adimensionalization, system (1) has been analysed in [3] and the main results are here reported for the convenience of the reader. Specifically, the following equilibria have been found: the origin, the predator-free equilibrium, two one predator-free points and the coexistence of the whole ecosystem. Together with these results, local stability and boundedness of the trajectories represent other relevant findings. 


\section{New findings on the non-delayed model}

We now proceed to extend the analysis. To this end, we need a slightly different adimensionalization of (1) than the one used in [3]. We namely introduce dimensionless variables into the model (1), as follows.

$$
P=\frac{p}{K}, \quad S_{\omega}=\frac{a s_{\omega}}{r}, \quad S_{c}=\frac{b s_{c}}{r}, \quad t=r T
$$

to get the following dimensionless version of the system of equations,

$$
\begin{aligned}
\frac{d P}{d t} & =P(1-P)-P S_{\omega}-P S_{c}, \\
\frac{d S_{\omega}}{d t} & =\alpha S_{\omega} P-\delta_{\omega} S_{\omega}-\frac{S_{\omega}^{2}}{W_{1}}, \\
\frac{d S_{c}}{d t} & =\beta S_{c} P-\delta_{c} S_{c}-\frac{S_{c}^{2}}{V_{1}},
\end{aligned}
$$

with once again feasible initial conditions, $P(0)>0, S_{\omega}(0)>0, S_{c}(0)>0$. The new introduced parameters are given by

$$
\alpha=\frac{e a K}{r}, \quad \beta=\frac{e b K}{r}, \quad \delta_{\omega}=\frac{\mu_{\omega}}{r}, \quad \delta_{c}=\frac{\mu_{c}}{r}, \quad W_{1}=a W, \quad V_{1}=b V .
$$

Two interesting dynamical properties which have not been addressed in [3], namely persistence of solutions originating from an interior point of the first octant and the global stability of the interior equilibrium are still unexplored and are investigated here.

\subsection{Equilibrium points and persistence}

The system has the following five equilibrium points: the origin $E_{0}$; the boundary points, in which the notation empasizes the nonvanishing components, $E_{1} \equiv(1,0,0)$;

$$
E_{12} \equiv\left(\frac{1+W_{1} \delta_{\omega}}{\alpha W_{1}+1}, \frac{\left(\alpha-\delta_{\omega}\right) W_{1}}{\alpha W_{1}+1}, 0\right) ; \quad E_{13} \equiv\left(\frac{1+V_{1} \delta_{c}}{\beta V_{1}+1}, 0, \frac{\left(\beta-\delta_{c}\right) V_{1}}{\beta V_{1}+1}\right)
$$

and the interior equilibrium point $E_{*} \equiv\left(P^{*}, S_{\omega}^{*}, S_{c}^{*}\right)$ where

$$
\begin{aligned}
P^{*}=\frac{1+\delta_{\omega} W_{1}+\delta_{c} V_{1}}{1+\alpha W_{1}+\beta V_{1}}, \quad S_{\omega}^{*} & =\frac{\left[\alpha\left(1+\delta_{c} V_{1}\right)-\delta_{\omega}\left(1+\beta V_{1}\right)\right] W_{1}}{1+\alpha W_{1}+\beta V_{1}}, \\
S_{c}^{*} & =\frac{\left[\beta\left(1+\delta_{\omega} W_{1}\right)-\delta_{c}\left(\alpha W_{1}+1\right)\right] V_{1}}{1+\alpha W_{1}+\beta V_{1}} .
\end{aligned}
$$


For feasibility of $E_{12}$ and $E_{13}$, we need respectively the restrictions on the parameters

$$
\alpha>\delta_{\omega}, \quad \beta>\delta_{c},
$$

while for the interior equilibrium we must require

$$
\alpha\left(1+\delta_{c} V_{1}\right)>\delta_{\omega}\left(1+\beta V_{1}\right), \quad \beta\left(1+\delta_{\omega} W_{1}\right)>\delta_{c}\left(\alpha W_{1}+1\right) .
$$

Persistence for a dynamical population system means that all its trajectories originating from an interior point of the positive orthant, in our case for (2) it is $\mathbb{R}_{+}^{3}$, remain strictly positive and do not approach any boundary of the non-negative cone as $t \rightarrow+\infty$. This implies the survival of all the populations for all future times. We now prove persistence of (2) by means of the well known "average Lyapunov function" technique, [5], which has been applied to a wide range of population models $[2,7,18]$.

Theorem 1. The system (2)-(4) is persistent if (5) hold together with

$$
\max \left\{\frac{\left(\alpha \delta_{c}-\beta \delta_{\omega}\right) W_{1}}{\beta-\delta_{c}}, \frac{\left(\beta \delta_{\omega}-\alpha \delta_{c}\right) V_{1}}{\alpha-\delta_{\omega}}\right\}<1 .
$$

Proof: For $\left(P, S_{\omega}, S_{c}\right) \in \mathbb{R}_{+}^{3}$, consider the positive definite Lyapunov function of the form

$$
\psi\left(P, S_{\omega}, S_{c}\right)=P^{\alpha_{1}} S_{\omega}^{\alpha_{2}} S_{c}^{\alpha_{3}}
$$

where $\alpha_{i}>0, i=1,2,3$ are three positive real numbers.

Calculating the logarithmic time derivative of $\psi(\cdot)$ along solution trajectories of the system (2) we get

$$
\frac{\dot{\psi}}{\psi}=\alpha_{1}\left[(1-P)-S_{\omega}-S_{c}\right]+\alpha_{2}\left[\alpha P-\delta_{\omega}-\frac{S_{\omega}}{W_{1}}\right]+\alpha_{3}\left[\beta P-\delta_{c}-\frac{S_{c}}{V_{1}}\right] .
$$

To prove the system to be persistent we need to show that the above logarithmic derivative is positive at all boundary equilibrium points $E_{0}, E_{1}, E_{12}, E_{13}$ for suitable choices of positive $\alpha_{i}$ 's.

The condition is ensured at the origin by the choice $\alpha_{1}>\alpha_{2} \delta_{\omega}+\alpha_{3} \delta_{c}$ and for the boundary equilibria $E_{1}, E_{12}$ and $E_{13}$ respectively, the following conditions need to be satisfied:

$$
E_{1}: \alpha_{2}\left(\alpha-\delta_{\omega}\right)+\alpha_{3}\left(\beta-\delta_{c}\right)>0,
$$




$$
\begin{aligned}
& E_{12}: \frac{\left(\alpha \delta_{c}-\beta \delta_{\omega}\right) W_{1}}{\beta-\delta_{c}}<1, \\
& E_{13}: \frac{\left(\beta \delta_{\omega}-\alpha \delta_{c}\right) V_{1}}{\alpha-\delta_{\omega}}<1 .
\end{aligned}
$$

But these are an easy consequence of the theorem assumptions.

\subsection{Global stability}

In this subsection first we prove that the interior equilibrium point $E_{*}$ is locally asymptotically stable whenever it exists. We calculate the Jacobian matrix for the system (2)-(4) at the interior equilibrium point finding

$$
J_{*}=\left[\begin{array}{ccc}
-P^{*} & -P^{*} & -P^{*} \\
\alpha S_{\omega}^{*} & -\frac{S_{\omega}^{*}}{W_{1}} & 0 \\
\beta S_{c}^{*} & 0 & -\frac{S_{c}^{*}}{V_{1}}
\end{array}\right]
$$

The characteristic equation for the matrix $J_{*}$ is given by

$$
\lambda^{3}+A_{1} \lambda^{2}+A_{2} \lambda+A_{3}=0,
$$

where

$$
\begin{aligned}
& A_{1}=P_{+}^{*} \frac{S_{\omega}^{*}}{W_{1}}+\frac{S_{c}^{*}}{V_{1}}>0, \\
& A_{2}=\left(1+\alpha W_{1}\right) \frac{P^{*} S_{\omega}^{*}}{W_{1}}+\left(1+\beta V_{1}\right) \frac{P^{*} S_{c}^{*}}{V_{1}}+\frac{S_{\omega}^{*} S_{c}^{*}}{W_{1} V_{1}}>0, \\
& A_{3}=\left(1+\alpha W_{1}+\beta V_{1}\right) \frac{P^{*} S_{\omega}^{*} S_{c}^{*}}{W_{1} V_{1}}>0 .
\end{aligned}
$$

According to the Routh-Hurwitz criteria [9], the interior equilibrium is locally asymptotically stable if $A_{1}>0, A_{3}>0$ and $A_{1} A_{2}>A_{3}$. After some algebraic calculations $A_{1} A_{2}-A_{3}>0$ can be verified, whenever $E_{*}$ is ecologically feasible.

We now show that the coexistence equilibrium is a global attractor, by constructing a suitable Lyapunov function. In fact, let us consider the following Lyapunov function,

$V(t)=\left[P-P^{*}-P^{*} \ln \frac{P}{P^{*}}\right]+A_{2}\left[S_{\omega}-S_{\omega}^{*}-S_{\omega}^{*} \ln \frac{S_{\omega}}{S_{\omega}^{*}}\right]+A_{3}\left[S_{c}-S_{c}^{*}-S_{c}^{*} \ln \frac{S_{c}}{S_{c}^{*}}\right]$ 
where $A_{2}$ and $A_{3}$ are two positive constants to be defined later. Taking the time derivative of $V(t)$ along solution curves of (2) we have,

$$
\begin{aligned}
\dot{V}= & {\left[(1-P)-S_{\omega}-S_{c}\right]\left(P-P^{*}\right)+A_{2}\left[\alpha P-\delta_{\omega}-\frac{S_{\omega}}{W_{1}}\right]\left(S_{\omega}-S_{\omega}^{*}\right) } \\
& +A_{3}\left[\beta P-\delta_{c}-\frac{S_{c}}{V_{1}}\right]\left(S_{c}-S_{c}^{*}\right) .
\end{aligned}
$$

Using

$$
1=P^{*}+S_{\omega}^{*}+S_{c}^{*}, \quad \delta_{\omega}=\alpha P^{*}-\frac{S_{\omega}^{*}}{W_{1}}, \quad \delta_{c}=\beta P^{*}-\frac{S_{c}^{*}}{V_{1}},
$$

the above expression can be rewritten as

$$
\begin{aligned}
\dot{V}= & -\left[\left(P-P^{*}\right)^{2}+\frac{A_{2}}{W_{1}}\left(S_{\omega}-S_{\omega}^{*}\right)^{2}+\frac{A_{3}}{V_{1}}\left(S_{c}-S_{c}^{*}\right)^{2}\right] \\
& -\left(P-P^{*}\right)\left[\left(S_{\omega}-S_{\omega}^{*}\right)+\left(S_{c}-S_{c}^{*}\right)-A_{2} \alpha\left(S_{\omega}-S_{\omega}^{*}\right)-A_{3} \beta\left(S_{c}-S_{c}^{*}\right)\right] .
\end{aligned}
$$

Taking then $A_{2}=\alpha^{-1}>0$ and $A_{3}=\beta^{-1}>0$ we have

$$
\dot{V}=-\left[\left(P-P^{*}\right)^{2}+\frac{1}{\alpha W_{1}}\left(S_{\omega}-S_{\omega}^{*}\right)^{2}+\frac{1}{\beta V_{1}}\left(S_{c}-S_{c}^{*}\right)^{2}\right]<0 .
$$

Now the set defined by $\left\{X \in \mathbb{R}^{3}:[d V / d t]_{X}=0\right\}$ consists of a single point $E_{*}$ and hence by La Salles's theorem [6], $E_{*}$ is globally asymptotically stable whenever it exists.

Combining the above results we can conclude that the destabilization of the coexisting equilibrium by a Hopf bifurcation is not possible once the parametric restrictions required for the existence of $E_{*}$ are satisfied. This also eliminates the chance of oscillatory coexistence.

\section{The delayed system}

In this Section we analytically prove persistence, global stability and the existence of a Hopf bifurcation for the model with delay, the latter being introduced due to biological reasons. In fact predators need time for the food consumption, due to prey handling and digesting. In particular webbuilding spiders store the captured prey, and therefore can survive for long times even without making new captures. 
The dimensionless form of the delayed version of (2-4) reads

$$
\begin{aligned}
\frac{d P(t)}{d t} & =P(t)(1-P(t))-P(t) S_{\omega}(t)-P(t) S_{c}(t), \\
\frac{d S_{\omega}(t)}{d t} & =\alpha S_{\omega}(t-\tau) P(t-\tau)-\delta_{\omega} S_{\omega}(t)-\frac{S_{\omega}^{2}(t)}{W_{1}} \\
\frac{d S_{c}(t)}{d t} & =\beta S_{c}(t-\tau) P(t-\tau)-\delta_{c} S_{c}(t)-\frac{S_{c}^{2}(t)}{V_{1}}
\end{aligned}
$$

subject to the initial conditions $P(\theta)=\phi_{1}(\theta)>0, S_{\omega}(\theta)=\phi_{2}(\theta)>0$, $S_{c}(\theta)=\phi_{3}(\theta)>0, \theta \in[-\tau, 0]$ where $\phi_{r} \in C\left([-\tau, 0] \rightarrow \mathbb{R}_{+}\right),(r=1,2,3)$ are given functions.

\subsection{Positivity}

First we prove the positivity of solutions for the model (8)-(10). Formally integrating (8) we find the following result

$$
P(t)=P(0) e^{\left[\int_{0}^{t}\left\{1-P(\xi)-S_{\omega}(\xi)-S_{c}(\xi)\right\} d \xi\right]},
$$

which implies $P(t)>0$ for all $t$ whenever $P(0)>0$.

Now from (9) we can write

$$
S_{\omega}(t)=S_{\omega}(0) e^{-\int_{0}^{t}\left\{\delta_{\omega}+\frac{S_{\omega}(\xi)}{W_{1}}\right\} d \xi}+\alpha \int_{0}^{t} S_{\omega}(\xi-\tau) P(\xi-\tau) e^{-\int_{\xi}^{t}\left\{\delta_{\omega}+\frac{S_{\omega}(\eta)}{W_{1}}\right\} d \eta} d \xi
$$

In view of the positive initial conditions $P(t)>0$ and $S_{\omega}(t)>0$ for $t \in$ $[-\tau, 0]$, from the above expression we infer that $S_{\omega}(t)>0$ for $t \in[0, \tau]$. Repeating this argument in the successive intervals of length $\tau$, namely $[\tau, 2 \tau]$, $[2 \tau, 3 \tau]$ and so on we find that $S_{\omega}(t) \geq 0$ for all $t>0$. Again from equation (10) we have

$$
S_{c}(t)=S_{c}(0) e^{-\int_{0}^{t}\left\{\delta_{c}+\frac{S_{c}(\xi)}{V_{1}}\right\} d \xi}+\beta \int_{0}^{t} S_{c}(\xi-\tau) P(\xi-\tau) e^{-\int_{\xi}^{t}\left\{\delta_{c}+\frac{S_{c}(\eta)}{V_{1}}\right\} d \eta} d \xi
$$

Using similar arguments as above we easily establish that $S_{c}(t) \geq 0$ for $t>0$. Hence the interior of the first octant is an invariant set for the delayed model (8)-(10). 


\subsection{Persistence}

Now we establish persistence of the delayed model, using the positivity of the dependent variables. We need to recall the following two lemmas, whose proofs can be found in [4] and [15] respectively.

Lemma 1. If $a, b>0$ and $u^{\prime}(t) \leq(\geq) u(t)(b-a u(t))$, with $u\left(t_{0}\right)>0$, then

$$
\limsup _{t \rightarrow \infty} u(t) \leq \frac{b}{a} \quad\left(\liminf _{t \rightarrow \infty} u(t) \geq \frac{b}{a}\right)
$$

Lemma 2. Consider the following equation

$$
\frac{d u(t)}{d t}=A u(t-\tau)-B u(t)-C u^{2}(t)
$$

where $A, B, C, \tau>0$ and $u(t)>0$ for $t \in[-\tau, 0]$, then we have following two results:

$$
\lim _{t \rightarrow+\infty} u(t)=\frac{A-B}{C}, \text { if } A>B ; \quad \lim _{t \rightarrow+\infty} u(t)=0, \text { if } A<B .
$$

These two lemmas are used to prove the persistence result. As the dependent variables are positive, from (8) we have the following inequality,

$$
\frac{d P(t)}{d t} \leq P(t)(1-P(t))
$$

and using Lemma 1 we get

$$
\limsup _{t \rightarrow+\infty} P(t) \leq 1=: M_{1}^{P} .
$$

So, for arbitrary $\epsilon>0$ there exist a positive real number $T$ such that

$$
P(t) \leq M_{1}^{P}+\epsilon, \quad \forall \quad t \geq T .
$$

Then for all $t \geq T+\tau$ from (9) we find

$$
\frac{d S_{\omega}(t)}{d t} \leq \alpha\left(M_{1}^{P}+\epsilon\right) S_{\omega}(t-\tau)-\delta_{\omega} S_{\omega}(t)-\frac{S_{\omega}^{2}(t)}{W_{1}}
$$


and then using Lemma 2 if $\alpha\left(M_{1}^{P}+\epsilon\right)>\delta_{\omega}$ we have

$$
\limsup _{t \rightarrow+\infty} S_{\omega}(t) \leq W_{1}\left(\alpha\left(M_{1}^{P}+\epsilon\right)-\delta_{\omega}\right)
$$

As $\epsilon>0$ is arbitrary, we can write

$$
\limsup _{t \rightarrow+\infty} S_{\omega}(t) \leq W_{1}\left(\alpha M_{1}^{P}-\delta_{\omega}\right)=: M_{1}^{S_{\omega}}
$$

whenever $\alpha M_{1}^{P}>\delta_{\omega}$. Using a similar argument from (10) we obtain whenever $\beta M_{1}^{P}>\delta_{c}$

$$
\limsup _{t \rightarrow+\infty} S_{c}(t) \leq V_{1}\left(\beta M_{1}^{P}-\delta_{c}\right)=: M_{1}^{S_{c}}
$$

On the other hand, using upper bounds for $S_{\omega}$ and $S_{c}$, from (8) for an arbitrary $\epsilon>0$, we get

$$
\frac{d P(t)}{d t} \geq P(t)(1-P(t))-\left(M_{1}^{S_{\omega}}+\epsilon\right) P(t)-\left(M_{1}^{S_{c}}+\epsilon\right) P(t)
$$

for sufficiently large $t$. As $\epsilon>0$ is arbitrarily small, if $M_{1}^{S_{\omega}}+M_{1}^{S_{c}}<1$ it follows

$$
\liminf _{t \rightarrow+\infty} P(t) \geq 1-M_{1}^{S_{\omega}}-M_{1}^{S_{c}}=: m_{1}^{P} .
$$

Using the above result and for arbitrary $\epsilon>0$ and for $t>T_{1}$, where $T_{1}$ denotes a sufficiently large positive number, from (9) we have

$$
\frac{d S_{\omega}(t)}{d t} \geq \alpha\left(m_{1}^{P}-\epsilon\right) S_{\omega}(t-\tau)-\delta_{\omega} S_{\omega}(t)-\frac{S_{\omega}^{2}(t)}{W_{1}}
$$

Using Lemma 2, and the arbitrariness of $\epsilon>0$ we find

$$
\liminf _{t \rightarrow+\infty} S_{\omega}(t) \geq W_{1}\left(\alpha m_{1}^{P}-\delta_{\omega}\right)=: m_{1}^{S_{\omega}}
$$

if $\alpha m_{1}^{P}>\delta_{\omega}$. Similar arguments and the assumption $\beta m_{1}^{P}>\delta_{c}$ give

$$
\liminf _{t \rightarrow+\infty} S_{c}(t) \geq V_{1}\left(\beta m_{1}^{P}-\delta_{c}\right)=: m_{1}^{S_{c}} .
$$

Summarizing, we have established the following two results: 
Theorem 2. If $\max \left\{\frac{\delta_{\omega}}{\alpha}, \frac{\delta_{c}}{\beta}\right\}<1$ then any solution of (8)-(10) starting from the interior of the first octant satisfies the following inequalities:

$$
\begin{aligned}
\limsup _{t \rightarrow+\infty} P(t) & \leq 1=: M_{1}^{P}, \\
\limsup _{t \rightarrow+\infty} S_{\omega}(t) & \leq W_{1}\left(\alpha M_{1}^{P}-\delta_{\omega}\right)=: M_{1}^{S_{\omega}}, \\
\limsup _{t \rightarrow+\infty} S_{c}(t) & \leq V_{1}\left(\beta M_{1}^{P}-\delta_{c}\right)=: M_{1}^{S_{c}} .
\end{aligned}
$$

Theorem 3. If $M_{1}^{S_{\omega}}+M_{1}^{S_{c}}<1$ and $\max \left\{\frac{\delta_{\omega}}{\alpha}, \frac{\delta_{c}}{\beta}\right\}<m_{1}^{p}$ then any solution of (8)-(10) starting from the interior of the first octant satisfies the following inequalities:

$$
\begin{aligned}
& \liminf _{t \rightarrow+\infty} P(t) \geq 1-M_{1}^{S_{\omega}}-M_{1}^{S_{c}}=: m_{1}^{P}, \\
& \liminf _{t \rightarrow+\infty} S_{\omega}(t) \geq W_{1}\left(\alpha m_{1}^{P}-\delta_{\omega}\right)=: m_{1}^{S_{\omega}}, \\
& \liminf _{t \rightarrow+\infty} S_{c}(t) \geq V_{1}\left(\beta m_{1}^{P}-\delta_{c}\right)=: m_{1}^{S_{c}} .
\end{aligned}
$$

These results thus ensure persistence of the delayed system.

\subsection{Global stability}

Let us introduce a new parameter

$$
\rho \equiv \alpha W_{1}+\beta V_{1} .
$$

Theorem 4. If the interior equilibrium $E_{*}$ exists and the conditions of Theorems 2 and 3 are satisfied together with

$$
\rho<1
$$

then $E_{*}$ is a global attractor.

Proof. Let

$$
\begin{array}{ll}
I_{1}=\limsup _{t \rightarrow+\infty} P(t), & J_{1}=\liminf _{t \rightarrow+\infty} P(t), \\
I_{2}=\limsup _{t \rightarrow+\infty} S_{\omega}(t), & J_{2}=\liminf _{t \rightarrow+\infty} S_{\omega}(t) \\
I_{3}=\limsup _{t \rightarrow+\infty} S_{c}(t), & J_{3}=\liminf _{t \rightarrow+\infty} S_{c}(t) .
\end{array}
$$


Then by Theorems 2 and 3,

$$
\begin{array}{ccc}
I_{1} \leq M_{1}^{P}, & I_{2} \leq M_{1}^{S_{\omega}}, & I_{3} \leq M_{1}^{S_{c}} \\
J_{1} \geq m_{1}^{P}, & J_{2} \geq m_{1}^{S_{\omega}}, & J_{3} \geq m_{1}^{S_{c}} \\
I_{1} \leq M_{2}^{P}, & I_{2} \leq M_{2}^{S_{\omega}}, & I_{3} \leq M_{2}^{S_{c}}, \\
J_{1} \geq m_{2}^{P}, & J_{2} \geq m_{2}^{S_{\omega}}, & J_{3} \geq m_{2}^{S_{c}},
\end{array}
$$

where,

$$
\begin{gathered}
M_{2}^{P}=1-m_{1}^{S_{\omega}}-m_{1}^{S_{c}}, \quad M_{2}^{S_{\omega}}=W_{1}\left(\alpha M_{2}^{P}-\delta_{\omega}\right), \quad M_{2}^{S_{c}}=V_{1}\left(\beta M_{2}^{P}-\delta_{c}\right) ; \\
m_{2}^{P}=1-M_{2}^{S_{\omega}}-M_{2}^{S_{c}}, \quad m_{2}^{S_{\omega}}=W_{1}\left(\alpha m_{2}^{P}-\delta_{\omega}\right), \quad m_{2}^{S_{c}}=V_{1}\left(\beta m_{2}^{P}-\delta_{c}\right) .
\end{gathered}
$$

Iterating the procedure we obtain

$$
m_{n}^{P} \leq J_{1} \leq I_{1} \leq M_{n}^{P}, \quad m_{n}^{S_{\omega}} \leq J_{2} \leq I_{2} \leq M_{n}^{S_{\omega}}, \quad m_{n}^{S_{c}} \leq J_{3} \leq I_{3} \leq M_{n}^{S_{c}},
$$

where

$$
\begin{gathered}
M_{n}^{P}=1-m_{n-1}^{S_{\omega}}-m_{n-1}^{S_{c}}, \quad M_{n}^{S_{\omega}}=W_{1}\left(\alpha M_{n}^{P}-\delta_{\omega}\right), \quad M_{n}^{S_{c}}=V_{1}\left(\beta M_{n}^{P}-\delta_{c}\right) ;(13) \\
m_{n}^{P}=1-M_{n}^{S_{\omega}}-M_{n}^{S_{c}}, \quad m_{n}^{S_{\omega}}=W_{1}\left(\alpha m_{n}^{P}-\delta_{\omega}\right), \quad m_{n}^{S_{c}}=V_{1}\left(\beta m_{n}^{P}-\delta_{c}\right) .
\end{gathered}
$$

So we have,

$$
M_{n+1}^{P}=\left(1+W_{1} \delta_{\omega}+V_{1} \delta_{c}\right)\left(1-\alpha W_{1}-\beta V_{1}\right)+\left(\alpha W_{1}+\beta V_{1}\right)^{2} M_{n}^{P} .
$$

Noting that $M_{n}^{P} \geq P^{*}$ and $\alpha W_{1}+\beta V_{1}<1$ it then follows

$$
M_{n+1}^{P}-M_{n}^{P}=\left[1-\left(\alpha W_{1}+\beta V_{1}\right)^{2}\right] P^{*}-\left[1-\left(\alpha W_{1}+\beta V_{1}\right)^{2}\right] M_{n}^{P} \leq 0 .
$$

Thus the sequence $\left\{M_{n}^{P}\right\}$ is monotonically decreasing and bounded below, and this implies that $\lim _{n \rightarrow \infty} M_{n}^{P}$ exists. Also $\lim _{n \rightarrow \infty} M_{n}^{P}=P^{*}$. Similarly from (13) and (14) we get

$$
\begin{array}{ll}
\lim _{n \rightarrow \infty} M_{n}^{S_{\omega}}=S_{\omega}^{*}, & \lim _{n \rightarrow \infty} M_{n}^{S_{c}}=S_{c}^{*}, \\
\lim _{n \rightarrow \infty} m_{n}^{P}=P^{*}, & \lim _{n \rightarrow \infty} m_{n}^{S_{\omega}}=S_{\omega}^{*}, \quad \lim _{n \rightarrow \infty} m_{n}^{S_{c}}=S_{c}^{*} .
\end{array}
$$

Now, $\lim _{n \rightarrow \infty} m_{n}^{P} \leq J_{1} \leq I_{1} \leq \lim _{n \rightarrow \infty} M_{n}^{P} \Rightarrow I_{1}=J_{1}$, and similarly the results $I_{2}=J_{2}$ and $I_{3}=J_{3}$ follow. Hence every sequence starting from an interior point of the first quadrant converges to the interior equilibrium point $E_{*}$. This completes the proof of the theorem.

Remark. An immediate consequence of this result is that whenever the conditions of Theorems 2 and 3 and $\rho<1$ are satisfied the discrete time delay cannot alter the stability property of the delayed system. Hence, in this situation the time delay is harmless, and this holds independently of the delay size. 


\subsection{Hopf-bifurcation analysis}

It is well known that discrete delays can destabilize the systems equilibria. Here we investigate whether this destabilization is possible through Hopf bifurcations. For this purpose we perform the Hopf-bifurcation analysis around the coexistence equilibrium $E_{*}$, taking ' $\tau$ ' as our bifurcation parameter. To this end, let us introduce perturbations of the variables of the delayed system (8)-(10) around the coexistence equilibrium as follows: $x=P-P^{*}$, $y=S_{\omega}-S_{\omega}^{*}$ and $z=S_{c}-S_{c}^{*}$ such that $|x|,|y|,|z| \ll 1$. Linearizing we find

$$
\begin{aligned}
\frac{d x}{d t} & =-P^{*} x(t)-P^{*} y(t)-P^{*} z(t), \\
\frac{d y}{d t} & =-\left(\delta_{\omega}+\frac{2 S_{\omega}^{*}}{W_{1}}\right) y(t)+\alpha S_{\omega}^{*} x(t-\tau)+\alpha P^{*} y(t-\tau), \\
\frac{d z}{d t} & =-\left(\delta_{c}+\frac{2 S_{c}^{*}}{V_{1}}\right) z(t)+\beta S_{c}^{*} x(t-\tau)+\beta P^{*} z(t-\tau) .
\end{aligned}
$$

The characteristic equation of the linearized system is,

$$
P_{1}(\lambda)+P_{2}(\lambda) e^{-\lambda \tau}+P_{3}(\lambda) e^{-2 \lambda \tau}=0,
$$

where

$P_{1}(\lambda)=\lambda^{3}+A_{1} \lambda^{2}+A_{3} \lambda+A_{6}, \quad P_{2}(\lambda)=-\left(A_{2} \lambda^{2}+A_{4} \lambda+A_{7}\right), \quad P_{3}(\lambda)=A_{5} \lambda+A_{8}$.

The coefficients of the polynomials $P_{r}(\lambda), r=1,2,3$ are

$$
\begin{aligned}
& A_{1}=P^{*}+\left(\delta_{\omega}+\delta_{c}\right)+2\left(\frac{S_{\omega}^{*}}{W_{1}}+\frac{S_{c}^{*}}{V_{1}}\right), \quad A_{2}=(\alpha+\beta) P^{*} \\
& A_{3}=\left[\left(\delta_{\omega}+\delta_{c}\right)+2\left(\frac{S_{\omega}^{*}}{W_{1}}+\frac{S_{c}^{*}}{V_{1}}\right)\right] P^{*}+\left(\delta_{\omega}+\frac{2 S_{\omega}^{*}}{W_{1}}\right)\left(\delta_{c}+\frac{2 S_{c}^{*}}{V_{1}}\right), \\
& A_{4}=\left[\beta\left(\delta_{\omega}+\frac{2 S_{\omega}^{*}}{W_{1}}\right)+\alpha\left(\delta_{c}+\frac{2 S_{c}^{*}}{V_{1}}\right)+(\alpha+\beta) P^{*}-\left(\alpha S_{\omega}^{*}+\beta S_{c}^{*}\right)\right] P^{*} \\
& A_{5}=\alpha \beta\left(P^{*}\right)^{2}, \quad A_{6}=\left(\delta_{\omega}+\frac{2 S_{\omega}^{*}}{W_{1}}\right)\left(\delta_{c}+\frac{2 S_{c}^{*}}{V_{1}}\right) P^{*} \\
& A_{7}=\left[\beta\left(\delta_{\omega}+\frac{2 S_{\omega}^{*}}{W_{1}}\right)\left(P^{*}-S_{c}^{*}\right)+\alpha\left(\delta_{c}+\frac{2 S_{c}^{*}}{V_{1}}\right)\left(P^{*}-S_{\omega}^{*}\right)\right] P^{*} \\
& A_{8}=\left[\alpha \beta\left(P^{*}-S_{\omega}^{*}-S_{c}^{*}\right)\right]\left(P^{*}\right)^{2} .
\end{aligned}
$$


Theorem 5. Necessary and sufficient conditions for $E^{*}$ to be locally asymptotically stable for all $\tau \geq 0$ are: $E^{*}$ is stable in absence of time delay $\tau$; there is no purely imaginary root of the characteristic equation (18).

This situation is indeed possible for our system, but the proof is quite tedious. However the last theorem in the previous section can be considered as an alternative proof of our claim. We will further verify in Section 4.5 this result by means of a numerical example.

Next we try to find under what conditions the system undergoes a Hopf bifurcation near the coexistence equilibrium $E^{*}$. We take the discrete time delay $\tau$ as a bifurcation parameter. Putting $\lambda=\mu+i \omega$ into equation (18) and separating the real and imaginary parts we get,

$$
\begin{array}{r}
\mu^{3}-3 \mu \omega^{2}+A_{1}\left(\mu^{2}-\omega^{2}\right)+A_{3} \mu+A_{6}-e^{-\mu \tau}\left[A_{2}\left(\mu^{2}-\omega^{2}\right) \cos \omega \tau\right. \\
\left.+A_{4} \mu \cos \omega \tau+A_{7} \cos \omega \tau+2 A_{2} \mu \omega \sin \omega \tau+A_{4} \omega \sin \omega \tau\right] \\
+e^{-2 \mu \tau}\left[A_{5} \mu \cos 2 \omega \tau+A_{8} \cos 2 \omega \tau+A_{5} \omega \sin 2 \omega \tau\right]=0, \\
3 \mu^{2} \omega-\omega^{3}+2 A_{1} \mu \omega+A_{3} \omega-e^{-\mu \tau}\left[2 A_{2} \mu \omega \cos \omega \tau+A_{4} \omega \cos \omega \tau\right. \\
\left.-A_{2}\left(\mu^{2}-\omega^{2}\right) \sin \omega \tau-A_{7} \sin \omega \tau-A_{4} \mu \sin \omega \tau\right] \\
+e^{-2 \mu \tau}\left[A_{5} \omega \cos 2 \omega \tau-A_{8} \sin 2 \omega \tau-A_{5} \mu \sin 2 \omega \tau\right]=0 .
\end{array}
$$

The necessary condition for a change of stability of the equilibrium point is that one of the characteristic roots is purely imaginary. Let $\tau=\widehat{\tau}$ be the particular magnitude of for which $\mu(\widehat{\tau})=0$ and $\omega(\widehat{\tau})=\widehat{\omega}>0$. Substituting $\tau=\widehat{\tau}, \mu=0$ and $\omega=\widehat{\omega}$ in (7) we get

$$
\begin{array}{r}
-\widehat{\omega}^{3}-A_{2} \widehat{\omega}^{2} \sin \widehat{\omega} \widehat{\tau}+A_{3} \widehat{\omega}-A_{4} \widehat{\omega} \cos \widehat{\omega} \widehat{\tau} \\
+A_{5} \widehat{\omega} \cos 2 \widehat{\omega} \widehat{\tau}+A_{7} \sin \widehat{\omega} \widehat{\tau}-A_{8} \sin 2 \widehat{\omega} \widehat{\tau}=0, \\
-A_{1} \widehat{\omega}^{2}+A_{2} \widehat{\omega}^{2} \cos \widehat{\omega} \widehat{\tau}-A_{4} \widehat{\omega} \sin \widehat{\omega} \widehat{\tau}+A_{5} \widehat{\omega} \sin 2 \widehat{\omega} \widehat{\tau}+A_{6} \\
-A_{7} \cos \widehat{\omega} \widehat{\tau}+A_{8} \cos 2 \widehat{\omega} \widehat{\tau}=0 .
\end{array}
$$

Eliminating $\cos 2 \widehat{\omega} \widehat{\tau}$ and $\sin 2 \widehat{\omega} \widehat{\tau}$, from the above system of equations we get

$$
\begin{aligned}
& \left(A_{3} \widehat{\omega}+A_{5} \widehat{\omega}-\widehat{\omega}^{3}\right) \cos \widehat{\omega} \widehat{\tau}+\left(A_{6}-A_{8}-A_{1} \widehat{\omega}^{2}\right) \sin \widehat{\omega} \widehat{\tau}=A_{4} \widehat{\omega} \\
& \left(A_{3} \widehat{\omega}-A_{5} \widehat{\omega}-\widehat{\omega}^{3}\right) \sin \widehat{\omega} \widehat{\tau}-\left(A_{6}+A_{8}-A_{1} \widehat{\omega}^{2}\right) \cos \widehat{\omega} \widehat{\tau}=A_{2} \widehat{\omega}^{2}-A_{7} .
\end{aligned}
$$

Solving (23) and (24) for $\cos \widehat{\omega} \widehat{\tau}$ and $\sin \widehat{\omega} \widehat{\tau}$, letting

$$
\Delta=A_{6}^{2}-2 A_{6} A_{1} \widehat{\omega}^{2}-A_{8}^{2}+A_{1}^{2} \widehat{\omega}^{4}+A_{3}^{2} \widehat{\omega}^{2}-2 A_{3} \widehat{\omega}^{4}-A_{5}^{2} \widehat{\omega}^{2}+\widehat{\omega}^{6},
$$


we get

$$
\begin{aligned}
\cos \widehat{\omega} \widehat{\tau}= & \frac{1}{\Delta}\left[A_{2} \widehat{\omega}^{2} A_{6}-A_{5} \widehat{\omega}^{2} A_{4}+A_{2} \widehat{\omega}^{4} A_{1}-\widehat{\omega}^{4} A_{4}\right. \\
& \left.-A_{7} A_{8}+A_{2} \widehat{\omega}^{2} A_{8}+A_{7} A_{6}+A_{3} \widehat{\omega}^{2} A_{4}-A_{7} A_{1} \widehat{\omega}^{2}\right], \\
\sin \widehat{\omega} \widehat{\tau}= & -\frac{1}{\Delta}\left[A_{2} \widehat{\omega}^{4}+\widehat{\omega}^{2} A_{4} A_{1}-\widehat{\omega}^{2} A_{3} A_{2}-\widehat{\omega}^{2} A_{5} A_{2}\right. \\
& \left.-\widehat{\omega}^{2} A_{7}+A_{5} A_{7}+A_{3} A_{7}-A_{4} A_{6}-A_{4} A_{8}\right] \widehat{\omega} .
\end{aligned}
$$

By squaring and adding the above two equations, we get a sixth degree algebraic equation in $\zeta=\widehat{\omega}^{2}$ with very complicated coefficients, which we omit. The required value of $\widehat{\omega}$ is the positive square root of the positive root of this sixth degree equation. The critical magnitude for the delay parameter is given by

$$
\widehat{\tau}=\frac{1}{\widehat{\omega}} \arcsin \left[\frac{\left(A_{3} \widehat{\omega}+A_{5} \widehat{\omega}-\widehat{\omega}^{3}\right)\left(A_{2} \widehat{\omega}^{2}-A_{7}\right)+A_{4} \widehat{\omega}\left(A_{6}+A_{8}-A_{1} \widehat{\omega}^{2}\right)}{\left(A_{3} \widehat{\omega}-\widehat{\omega}^{3}\right)^{2}+\left(A_{6}-A_{1} \widehat{\omega}^{2}\right)^{2}-A_{5}^{2} \widehat{\omega}^{2}-A_{8}^{2}}\right] .
$$

The transversality condition for the Hopf bifurcation at $\tau=\widehat{\tau}$ is $\left[\frac{d \mu}{d \tau}\right]_{\tau=\widehat{\tau}} \neq$ 0 . Differentiating (7a) and (7b) with respect to $\tau$ and putting $\tau=\widehat{\tau}, \omega=\widehat{\omega}$, $\mu=0$, we get

$$
E\left[\frac{d \mu}{d \tau}\right]_{\tau=\widehat{\tau}}+F\left[\frac{d \omega}{d \tau}\right]_{\tau=\widehat{\tau}}=G, \quad-F\left[\frac{d \mu}{d \tau}\right]_{\tau=\widehat{\tau}}+E\left[\frac{d \omega}{d \tau}\right]_{\tau=\widehat{\tau}}=H
$$

where

$$
\begin{aligned}
E= & -3 \widehat{\omega}^{2}+A_{3}-A_{2} \widehat{\omega}^{2} \widehat{\tau} \cos \widehat{\omega} \widehat{\tau}+A_{7} \widehat{\tau} \cos \widehat{\omega} \widehat{\tau}+A_{4} \widehat{\omega} \widehat{\tau} \sin \widehat{\omega} \widehat{\tau}-A_{4} \cos \widehat{\omega} \widehat{\tau} \\
& -2 A_{2} \widehat{\omega} \sin \widehat{\omega} \widehat{\tau}-2 A_{8} \widehat{\tau} \cos 2 \widehat{\omega} \widehat{\tau}-2 A_{5} \widehat{\omega} \widehat{\tau} \sin 2 \widehat{\omega} \widehat{\tau}+A_{5} \cos \widehat{\omega} \widehat{\tau} \\
F= & -2 A_{1} \widehat{\omega}+2 A_{2} \widehat{\omega} \cos \widehat{\omega} \widehat{\tau}-A_{2} \widehat{\omega}^{2} \widehat{\tau} \sin \widehat{\omega} \widehat{\tau}+A_{7} \widehat{\tau} \sin \widehat{\omega} \widehat{\tau}-A_{4} \sin \widehat{\omega} \widehat{\tau} \\
& -A_{4} \widehat{\omega} \widehat{\tau} \cos \widehat{\omega} \widehat{\tau}-2 A_{8} \widehat{\tau} \sin 2 \widehat{\omega} \widehat{\tau}+A_{5} \sin 2 \widehat{\omega} \widehat{\tau}+2 A_{5} \widehat{\omega} \widehat{\tau} \cos 2 \widehat{\omega} \widehat{\tau}, \\
G= & A_{2} \widehat{\omega}^{3} \sin \widehat{\omega} \widehat{\tau}-A_{7} \widehat{\omega} \sin \widehat{\omega} \widehat{\tau}+A_{4} \widehat{\omega}^{2} \cos \widehat{\omega} \widehat{\tau}+2 A_{8} \widehat{\omega} \sin 2 \widehat{\omega} \widehat{\tau}-2 A_{5} \widehat{\omega}^{2} \cos 2 \widehat{\omega} \widehat{\tau}, \\
H= & A_{2} \widehat{\omega}^{3} \cos \widehat{\omega} \widehat{\tau}-A_{7} \widehat{\omega} \cos \widehat{\omega} \widehat{\tau}-A_{4} \widehat{\omega}^{2} \sin \widehat{\omega} \widehat{\tau}+2 A_{8} \widehat{\omega} \cos 2 \widehat{\omega} \widehat{\tau}+2 A_{5} \widehat{\omega}^{2} \sin 2 \widehat{\omega} \widehat{\tau} .
\end{aligned}
$$

Thus we have

$$
\left[\frac{d \mu}{d \tau}\right]_{\tau=\widehat{\tau}}=\frac{G E-H F}{E^{2}+F^{2}}
$$

But from this expression it is quite difficult to assess whether the transversality condition is satisfied. We verify it only numerically in the next Section. 


\subsection{Numerical simulations}

Now we can verify the above results numerically. For this purpose we choose two sets of parameter values. Using the former we prove that the delay cannot destabilize the coexistence equilibrium. The second set shows a Hopf-bifurcating periodic solution originating as the discrete delay crosses the threshold magnitude.

In the first case consider these parameter values: $\alpha=.6, \beta=.8, \delta_{\omega}=.1$, $\delta_{c}=.2, W_{1}=.2, V_{1}=1$, for which the interior equilibrium point of the non-delayed model is globally asymptotically stable and all other axial and boundary equilibria are saddle points. In Fig. 1 the unstable equilibrium points are denoted by black circles and the globally stable interior equilibrium point is marked with a red-filled circle. For the origin there are two stable manifolds, the deep green lines. Its unstable manifold, the blue curve, connects it with $E_{1}$. The two unstable manifolds of $E_{1}$, the cyan curves, converge to the boundary equilibria $E_{12}$ and $E_{13}$. The two unstable manifolds of $E_{12}$ and $E_{13}$, the violet curves, connect them with the interior equilibrium point. Another violet curve coming from above and converging to the interior equilibrium point lies on the center manifold of $E_{*}$; two eigenvalues of $E_{*}$ are complex conjugate with negative real parts for the chosen parameter values.

For the parameter values given above, the characteristic equations (23)(24) become

$\zeta^{6}+2.227852 \zeta^{5}+2.239491 \zeta^{4}+1.269975 \zeta^{3}+.424629 \zeta^{2}+.07849 \zeta+0.006165=0$.

This equation does not have positive real roots and consequently the discrete delay parameter cannot alter the stability properties. In this case there is no delay-induced instability as the conditions of Theorems 2 and 3 and $\alpha W_{1}+\beta V_{1}=0.92<1$ are satisfied and hence $E_{*}$ is a global attractor for the delayed system also. The stable solution is presented in Fig 2 for a quite large value of the delay.

Next we consider the following parameters: $\alpha=2, \beta=3, \delta_{\omega}=.1, \delta_{c}=.2$, $W_{1}=2.5, V_{1}=2$. For this choice of parameter values the equations (23)-(24) become

$\zeta^{6}+.751406 \zeta^{5}+.178689 \zeta^{4}+.020433 \zeta^{3}+.000141 \zeta^{2}-.000955 \zeta-.000133=0$.

This has only one positive root $\zeta=.1578675228$. Thus in this case the system undergoes a Hopf bifurcation for $\tau=1.913741915$. Since the above 


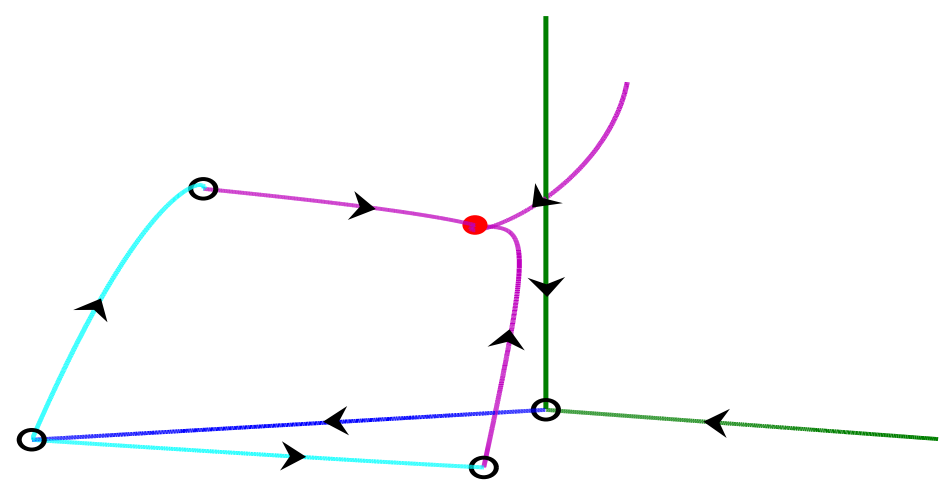

Figure 1: The stable and unstable manifolds of all equilibria are presented here, see the text for details. The interior equilibrium point, filled and coloured in red, is the only attractor lying in the interior of the positive octant.

equation possesses only one positive root, and hence there is no possibility of switching of stability. The Hopf-bifurcating periodic solution is in fact presented in Fig 3.

In this situation one may ask what happens if the magnitude of delay parameter increases beyond the Hopf-bifurcation threshold. To answer this question we have investigated numerically the delayed model with a second set of parameter values $\alpha=2, \beta=3, \delta_{\omega}=.1, \delta_{c}=.2, W_{1}=2, V_{1}=2.5$, keeping $\tau$ as free parameter. The chosen set of parameter values satisfy all necessary criteria for the persistence of all species and hence extinction of any species is not possible even for large values of the delay parameter. The model undergoes a period doubling route to chaos with increasing magnitudes of the delay parameter. Projection of the three dimensional attractors on the $P-S_{w}$-plane is presented in Fig. 4 for four different values of $\tau$ depicting the period doubling scenario. Projection of chaotic attractor on $\ln (P)-S_{w}$-plane is given in Fig. 5, logarithmic scale on $P$ axis is chosen for clear visualization. Sensitivity to initial condition is checked to ensure the chaotic nature of the attractor obtained for $\tau=30$. The divergence of two nearby trajectories, 


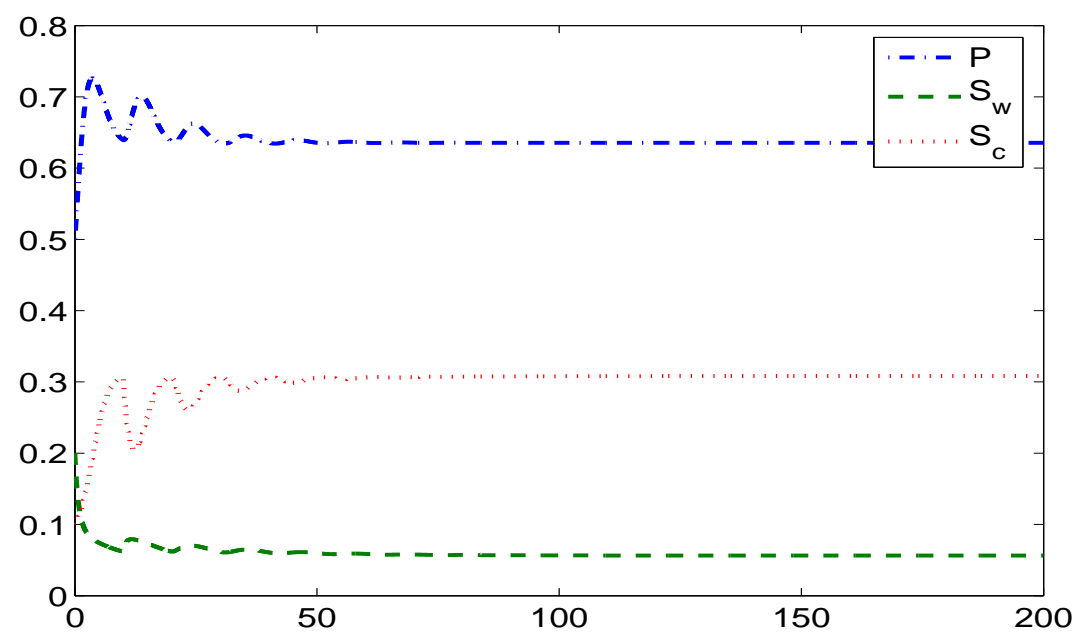

Figure 2: Stable solution for $\tau=10$. Top blue dash-dotted curve for the prey, bottom green dashed for the first spider population and middle red dotted curve for the second spider population.

another signature of chaos, is presented in Fig. 6. The divergence of $P(t)$ for two solution trajectories is plotted against time. A bifurcation diagram, indicating chaotic dynamics for the prey population is presented in Fig. 7. Analogous ones arise for the predators.

\section{Discussion}

A nonlinear ordinary differential equation model and its corresponding delayed version ware proposed in [3] for an agro-ecosystem consisting of two spider populations and an insect pest population which serves as their prey. Here we have established the global stability of the coexistence equilibrium point for the non-delayed model together with the impossibility of oscillatory coexistence. We have derived the persistence conditions for both the non-delayed and delayed models. The criteria for global stability of the coexisting equilibrium point for the delayed model is derived without using any Lyapunov-like function. Recalling (11), if the criteria for global stability is satisfied the delay has no destabilizing effect. In order to capture the oscillatory coexistence we performed the Hopf-bifurcation analysis with discrete 


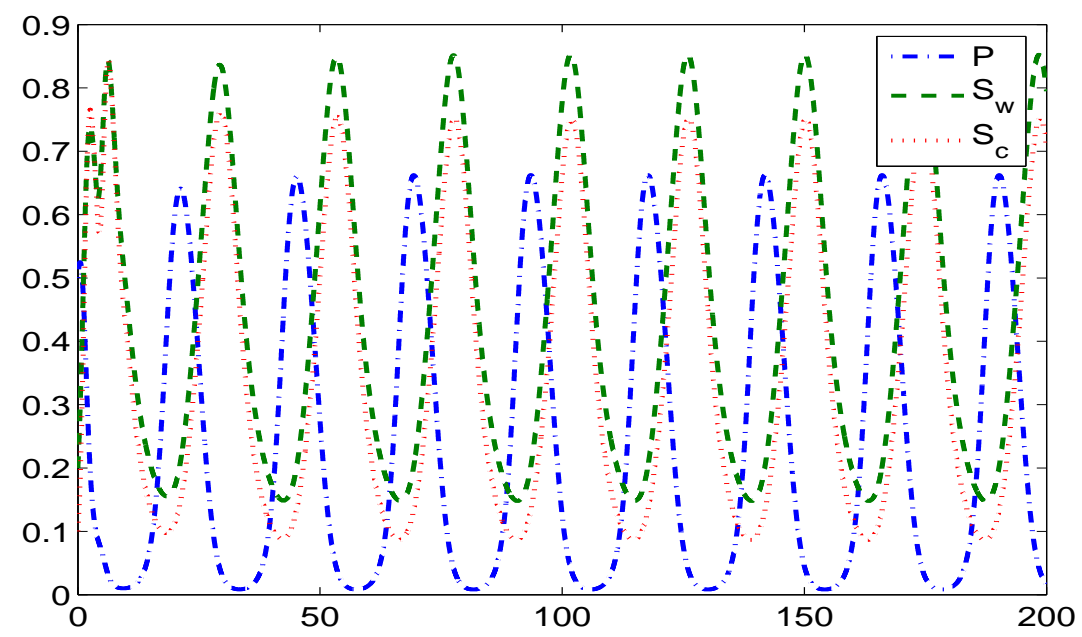

Figure 3: Hopf-bifurcating periodic solution for the three populations obtained for $\tau=4>\widehat{\tau}$. Blue dash-dotted curve for the prey, green dashed for the first spider population and red dotted curve for the second spider population

delay as bifurcation parameter and our analysis reveals that oscillatory coexistence is possible under certain circumstances. Hence the destabilizing role of the delay is related with the growth rates of spider populations and the strength of their intra-specific competition rates. With numerical investigation we have shown that once the threshold condition $\rho>1$ holds, persistent large amplitude oscillations as well as chaotic coexistence become possible. Satisfaction of the conditions in Theorems 2 and 3 for chosen parameter values ensure the persistence of all species.

The interpretation of the threshold parameter $\rho$ is as follows. If we ignore spiders' mortalities, i.e. setting $\delta_{c} \approx 0, \delta_{\omega} \approx 0$, indicating with bars the population levels at the coexistence equilibrium in this case, we find that (6) becomes trivially satisfied. Moreover, $\bar{S}_{\omega}=\alpha W_{1} \bar{P}, \bar{S}_{c}=\beta V_{1} \bar{P}$. Hence, indicating with $\bar{S}$ the total spiders population, we find

$$
\rho=\alpha W_{1}+\beta V_{1}=\frac{\bar{S}_{\omega}+\bar{S}_{c}}{\bar{P}}=\frac{\bar{S}}{\bar{P}} .
$$

Thus, ignoring spiders' mortality, $\rho$ is a measure of the fraction of the total spiders population versus the pest population at equilibrium. Thus, if 

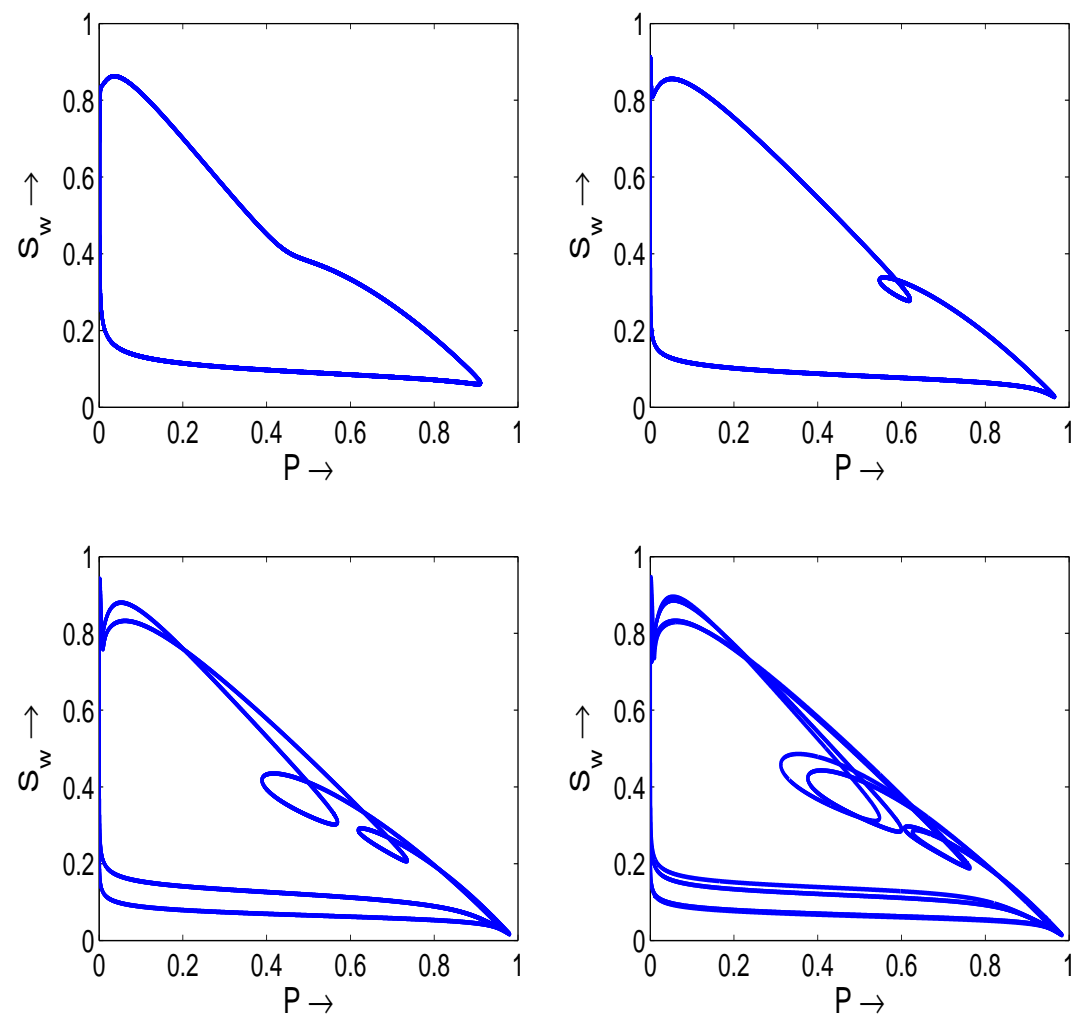

Figure 4: Projection of stable attractors on $P-S_{w}$-plane for $\tau=10$ (upper panel, left), $\tau=17$ (upper panel, right), $\tau=20$ (lower panel, left) and $\tau=21.5$ (lower panel, right). Stable attractors having period one, two, four and eight respectively. Other parameter values are mentioned in the text.

the latter exceed the predators, a stable equilibrium is attained also in the presence of delays. But conversely, the magnitude of the delay may trigger oscillations. In view of the fact that spiders are known to be able to survive long times of starvation, mortalities can be assumed to have values close to zero, thereby satisfying the assumption behind (27). Further, this implies also that the prey is stored for long times, making its handling time likely to attain large values. Thus the influence of the delay parameter becomes of paramount importance in this situation as it certainly triggers oscillations, corresponding to periodic outbreaks of the pests in the field. Our simula- 


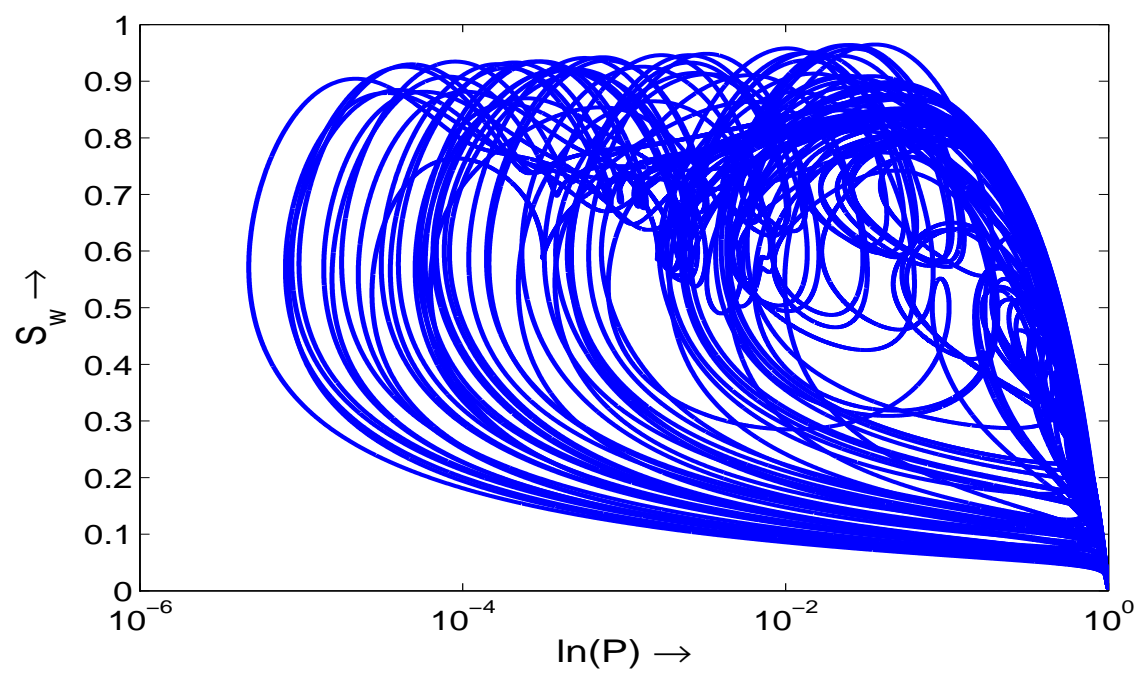

Figure 5: Projection of the chaotic attractor on $\ln (P)-S_{w}$-plane $(\tau=30)$.

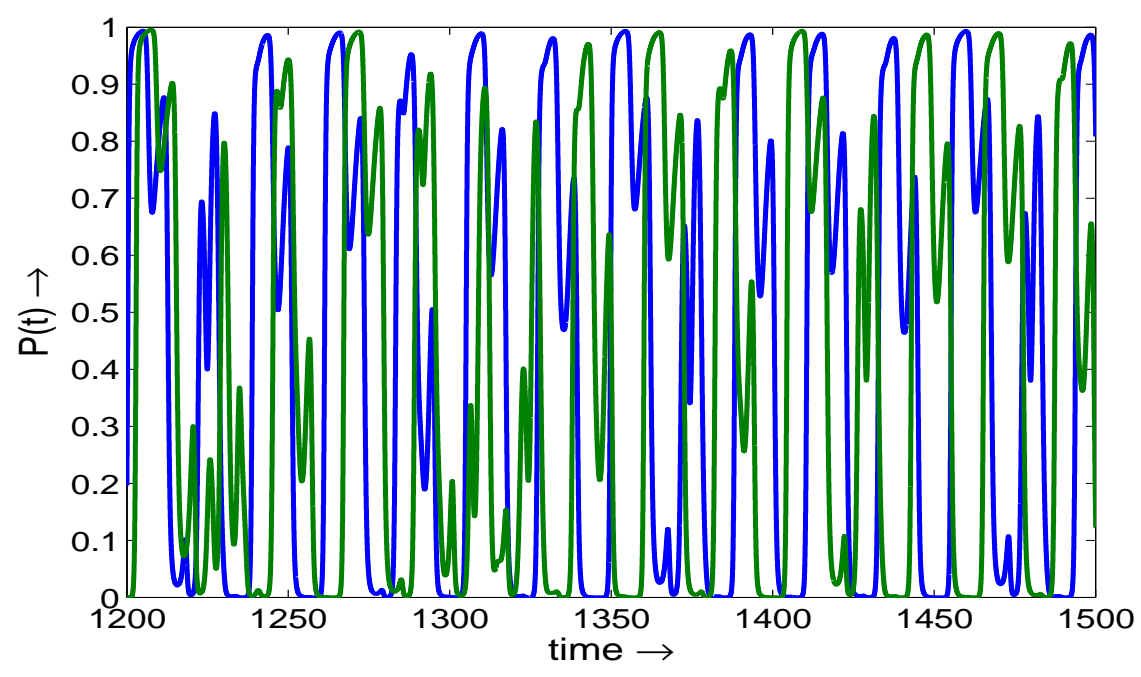

Figure 6: Two solution trajectories are obtained for a small deviation (of the order of $10^{-2}$ ) in the initial condition, and divergence of trajectories is captured through the divergence of the $P$ component.

tions, Figures 2 and 3, are based on hypothetical parameter values and show that the peaks of the oscillations are essentially at the same level as the pop- 


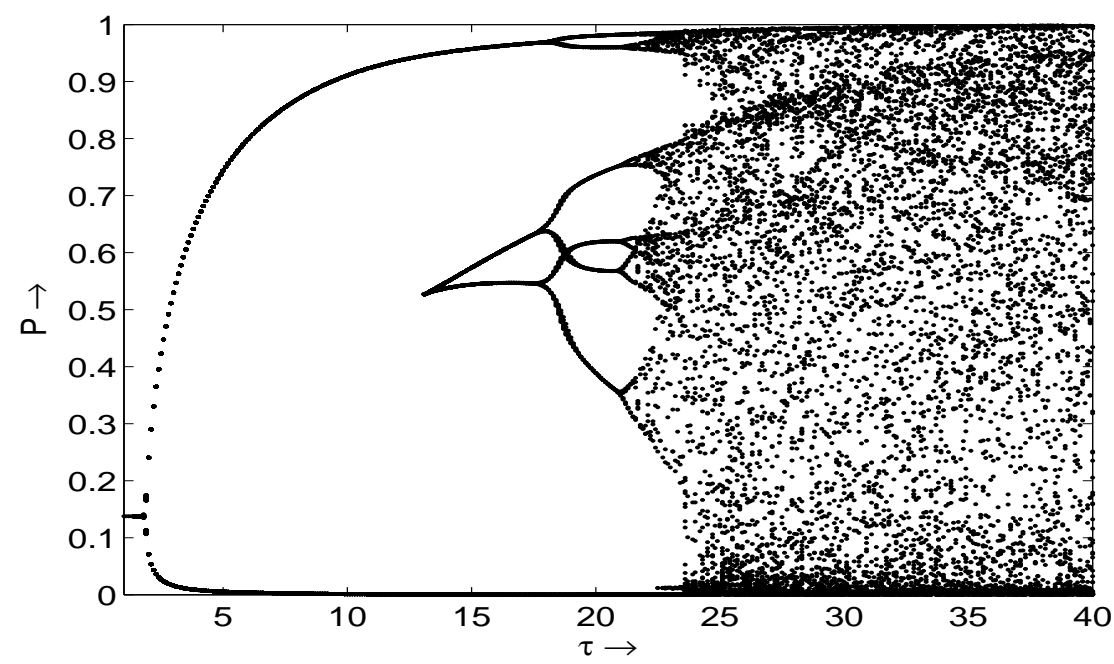

Figure 7: Bifurcation diagram for route to chaos with $\tau$ as bifurcation parameter. Here the local maximum and minimum of $P(t)$ are plotted after deleting some initial transients for a large range of values of the delay $\tau$.

ulations at steady state. In fact, the peaks for the prey in Figure 3 are lower than their equilibrium values in Figure 2. But in practical situations, the result depends on the model parameter values. It could be possible that the pest outbursts be much larger than the equilibrium levels. Note that in our situation also the assumption of Theorem 2 holds, since $\delta_{c} \approx 0, \delta_{\omega} \approx 0$, as well as the ones of Theorem 3, since the first one corresponds to (12). Since persistence of the system is thus ensured if (12) holds, to try to eradicate the pests one could think of artificially increasing the number of spiders. This measure should then destabilize the equilibrium, but as seen, in the presence of long delays in prey consumption it could lead to pests outbursts. If the latter much exceed their usual equilibrium value at steady state, one has to evaluate whether it is better to accept the pests equilibrium level, or to have most of the time the pests at low levels, paying the price of periodically experiencing large outbreaks of them. Finally it should be remarked that with these types of oscillations, there is the usual danger that environmental fluctuations may very well wipe out the populations that thrive at low levels in the troughs of these cycles, and this collapsing could include also spiders, thereby just worsening the situation. To assess the best course for the field 
manager is beyond the purpose of this paper, as it hinges on the values of the model parameters that need to be obtained by field measurements. Therefore the final outcome of the system and the possible recommendations for the course to take in order to control pests invasions depend ultimately on the particular situation at hand.

Acknowledgments: The work of first author [M.S.] is supported by the Council of Scientific and Industrial Research, India. The second author acknowledges the financial help from the D.S.T. New Delhi, India, under its research project SR/S4/MS:581/09.

\section{References}

[1] P. Batáry, A. Holzschuh, K. M. Orci, F. Samu, T. Tscharntke, Responses of plant, insect and spider biodiversity to local and landscape scale management intensity in cereal crops and grasslands, Agriculture, Ecosystems and Environment 146 (2012) 130 - 136.

[2] S. Chatterjee, M. Bandyopadhyay, J. Chattopadhyay, Proper predation makes the system disease free - conclusion drwan from an ecoepidemiological model, J. Biol. Syst. 14 (2006) 599 - 616.

[3] S. Chatterjee, M. Isaia, E. Venturino, Effects of spiders predational delays in extensive agroecosystems, Nonlin. Anal. RWA, 10 (2009) 3045 - 3058.

[4] F. D. Chen, Z. Li, X. Chen, L. Jitka, Dynamic behaviors of a delay differential equation model of plankton allelopathy, J. Comput. Appl. Math., 206 (2007) 733 - 754.

[5] T. C. Gard, T. G. Hallam, Persistence in food web-1, Lotka-Volterra food chains, Bull. Math. Bio. 41 (1979) 877 - 891.

[6] J. K. Hale, Ordinary Differential Equations, Wiley, New York, 1969.

[7] V. Hutson, G. T. Vickers, A criterion for permanent co-existence of species with an application to two prey, one predator system, Math. Biosci. 63 (1983) $253-269$. 
[8] L. M. Kerzicnik, Spiders as potential aphid predators in eastern Colorado agroecosystems, Ph.D. Dissertation, Colorado State University, 2011, 217 pages.

[9] J. D. Murray, Mathematical biology, Springer, Heidelberg (1989).

[10] N.N. Ntonifor, M.C. Parr and J.A. Ewunkem, Seasonal abundance and distribution of the huntsman spider, Heteropoda venatoria (Sparassidae: Araneae) in banana agro-ecosystems in Cameroon, Journal of Entomology 9 (2012) 79 - 88.

[11] S. Prieto-Benítez, M. Méndez, Effects of land management on the abundance and richness of spiders (Araneae): a meta-analysis, Biological Conservation 144 (2011) 683 - 691.

[12] E. N. L. Rodrigues, M. de S. Mendonça Jr., R. Ott, Spider diversity in a rice agroecosystem and adjacent areas in southern Brazil, Rev. Colomb. Entomol. 35(1) (2009) 89 - 97.

[13] R. Royauté, C. M. Buddle, Colonization dynamics of agroecosystem spider assemblages after snow-melt in Quebec (Canada), Journal of Arachnology 40(1) (2012) 48 - 58.

[14] R. R. Seyfulina, The spider assemblage (Arachnida, Aranei) in agroecosystems of the Kuban Plain: species composition, spatial distribution, and seasonal dynamics, Entomological Review 90(4) (2010) 494 - 510.

[15] X. Song, L. Chen, Optimal harvesting and stability for a two-species competitive system with stage structure, Math. Biosci. 170 (2001) 173 186.

[16] Venkateshalu, Hanumanthraya, L., Girish Maraddi, Impact of different rice agro-ecosystem on spider population dynamics, Environment and Ecology 27(3A) (2009) 1231 - 1236.

[17] E. Venturino, M. Isaia, F. Bona, E. Issoglio, V. Triolo, G. Badino, Modelling the spiders ballooning effect on the vineyard ecology, Math. Model. Nat. Phenom. 1 (2006) 137 - 159.

[18] P. Waltman, A brief survey of persistence in dynamical systems, in Delay differential equations and dynamical systems, S. Busenberg, M. Martelli 
(Editors), 'Lecture Notes in Mathematics, Vol. 1475', Springer, New York, 31 - 40, 1991. 